\title{
SVM based Signature Verification by Fusing Global and Functional Features
}

\author{
Biswajit Kar \\ Department of Electrical Engineering, IIT, \\ Kharagpur, India.
}

\author{
Pranab. K. Dutta \\ Department of Electrical Engineering, IIT, \\ Kharagpur, India.
}

\begin{abstract}
On-line signature verification can be used in real time applications like credit card transactions or resource accesses because of its popularity in regular authentication. In signature verification number of signatures avalible to train a model is very limited, and therefore identification of the most suitable features which characterize the class is critical. Therefore feature selection is essential to minimize the classification error. The mRMR (minimum Redundancy Maximum Relevance) method is applied to select the features. Verification is based on global features and scores from functional features. The scores are generated by comparing the functional features of the test signature with the corresponding reference features. These scores are treated as additional features in a two-class classification problem solved with the ANN and SVM. Verification accuracy is enhanced by fusion of user specific global and functional features. The methods are tested with the database of SVC2004.
\end{abstract}

\section{Keywords}

Support vector machine, On-line signature verification, Feature selection, mRMR.

\section{INTRODUCTION}

Automatic signature verification is a commonly used form of biometric verification and identification, because of wide spread acceptance of static signature in the application of personal authentication, document certification for a very long time in manual verification [1]. It can be predicted that as the technology enhances online signature will be one of the important means of biometric in this field with good user acceptance. The online context is more desirable to prevent imitation. An impostor can imitate visible shape of the signature, but it is nearly impossible to achieve the imitation of dynamic content of the signature, which is embed in the gesture of signing and is very personal.

\section{LITERATURE REVIEW}

One of the first publications on on-line signature verification was by Herbst and Liu [2]. In this paper handwriting was modeled as ballistic motions that do not involve sensory feedback. Extensive literature is available in the field of online (dynamic) signature verification. A survey of signature verification can be found in [3-5].

Leclerc and Plamondon categorized the various signature verification methodologies into two types: functional approach and parametric approach [4]. In the function-based approach, online signatures are characterized and analyzed as time sequences (e.g., position trajectory, velocity, acceleration, pressure, direction of pen movement, and azimuth) [6].

In general, function-based features show better discriminating ability than the parameter-based features but they usually require time-consuming algorithm for comparison. However, the work by Aguilar et al. reported that the parametric approaches are equally competitive with the function based approaches [7]. In parametric approaches, the authenticity of a test signature is estimated by comparing test feature set against reference feature set. Each matching method is based on similarity (or dissimilarity) measurement. In the parameter based approach, one commonly used distance measure is Euclidean distance [6]. The verification methods are based on Neural Networks (NN) [8], Hidden Markov Model (HMM) [9], and Support vector machine (SVM) are mostly used [10-11].

Function-based approaches can be classified into local and regional methods. In local approaches, the time functions of different signatures are directly matched by using elastic matching technique such as dynamic time warping [12-16]. However, the time complexity of DTW is of $O\left(n^{2}\right)$. In the case of function-based approach, the matching methods must take care of the phase shift and non-linear distortion of functions.

A popular technique used for signature verification is the SVM. With the help of training examples from two classes, an SVM search the maximum separating hyperplane. In [11], comparison of SVM classifiers with HMM classifiers is carried out in terms of the number of samples used for training and verification using different types of forgeries. Under both conditions, SVM appears to produce better result. However, the main limitations of SVMs are high algorithmic complexity and extensive memory requirements in large-scale tasks.

Signature verification schemes based on Neural Network are also proposed [8]. Although, the neural network-based approaches have the capabilities in generalization, the drawback is the need for a large number of genuine and forgery signatures for training, which is not always practically viable.

In the verification system user data should be described in such a way that it will discriminate the user maximally. To fulfill this purpose the intra class variability is to be reduced 
and at the same time the inter class variability is to be preserved. To reduce the intra class variation and keeping the discriminatory feature, it is better to normalize the signature in the function domain before feature extraction and matching. The fusion of function and feature based verification score gives better accuracy. Selection of the consistent statistical feature is a great challenge in feature based verification [17].

\section{ONLINE SIGNATURE VERIFICATION SYSTEM}

A diagram of a general signature verification system is shown in Fig. 2.
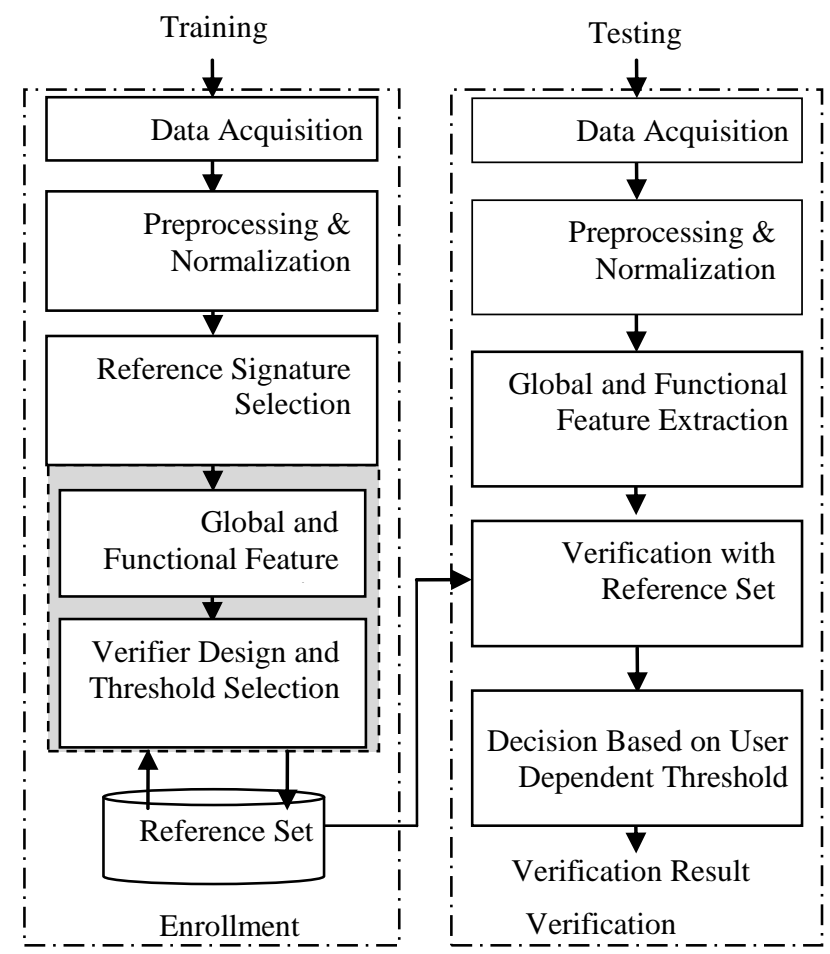

Figure 2: General model of online signature verification

Signature verification systems are generally divided into two modules: Signature enrollment or training module and signature verification or testing module. A signature verification system must provide a solution to the problems of data acquisition, preprocessing and normalization, reference signature selection, feature extraction, matching, and performance evaluation. Out of these feature selection and verification methods have been considered in this paper. Detail discussion of other modules is beyond the scope of this paper.

\section{DATABASE}

First international signature verification competition (SVC2004) was held as a step towards establishing common benchmark databases and benchmarking rules [18]. For each of the two tasks of the competition, a signature database involving 40 sets of signature data was created, with 20 genuine signatures and 20 skilled forgeries for each set. Experimental results have been conducted using the Task 1
SVC2004 database. Shape of online signature and its associated function are shown in Fig, 1.

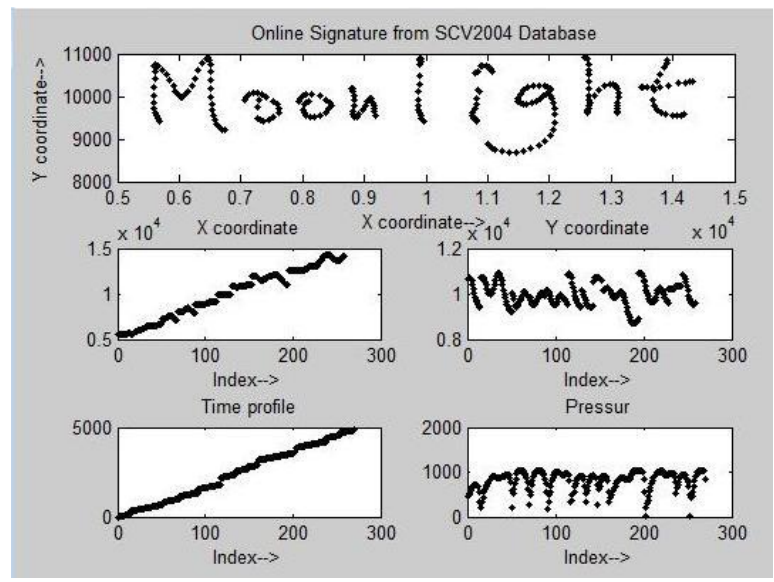

Figure 1: On-line signature shape and its associated functions from SVC2004 database

\section{FEATURE EXTRACTION}

Both global feature and functional feature are used here for verification.

\subsection{Global Feature Extraction}

In the global parametric approach, a fixed set of parameter is extracted to describe a signature pattern. More importantly, this approach is expected to be more stable against the variations in local regions, which are common in signatures. The difficulty with this approach lies in selecting the salient parameters that can distinguish between the classes and are consistent among the same set. The major limitation of this approach lies in its discriminative ability [19-20]. An averaging effect arises in calculating the parameters over the whole pattern. Although this effect is obviously the reason for the above-mentioned stability, the parameters selected from a small set of signers may not work well on a larger set of signers [19].

However, verification with global features of a signature has several advantages. It is simple to compute and address the concerns related to privacy because it does not need to retain the original signature once the features are extracted. In total 48 global features are calculated here for every signature.

\subsection{Functional Feature Extraction}

In the functional approach, complete signals $[x(i), y(i)$, $p(i), t(i)$ etc, where, $i$ is index of the signature samples] directly or indirectly constitute the feature set. The two signals, one from the reference signature and the other from the test signature, are then compared point-to-point or segment-to-segment basis [21]. The challenge within this approach is that two signals are likely to have different durations and also undergo non-linear distortions.

Seventeen signature functions (local features) are taken for score generation. Three measures are estimated for comparison, namely 1) correlation coefficient 2) DTW 
distance 3) Euclidean distance. From the outputs of three measures total $17 \times 3=51$ scores are estimated.

\section{FEATURE SELECTION}

In signature verification number of sample signature avalible to train a verifier model is very limited [22], whereas number of avalible features (or attributes) are very large compared to the sample data. Feature vector with very large dimensionality leads to th curse of dimensionality problem [23]. Identification of the most suitable features of the oserved data which characterize the class is also critical. Therefore feature selection is very essetial to minimize the inconsistancy in classification.

\subsection{Feature Selection by mRMR Method}

In an unsupervised situation minimal error usually requires the maximal statistical dependency of the target class, say C, on the data distribution [22, 24]. The method of Minimum Redundancy Maximum Relevance (mRMR) has been proposed by Peng et al. [22].

One of the most popular approaches to realize maximum dependency is maximum relevance feature selection, i.e., selecting the features with the highest relevance to the target class C. Relevance is usually characterized in terms of correlation or mutual information. Results of global feature selection by mRMR method are shown in Table 1 . Out of 48 features, 10 best selected features using mRMR method is shown in Table 1.

Table 1: 10 common selected features

\begin{tabular}{|c|c|c|}
\hline $\begin{array}{l}\text { Average } \\
\text { Rank }\end{array}$ & $\begin{array}{l}\text { Symbol } \\
\text { used }\end{array}$ & Name and description. \\
\hline 9.45 & $\mu_{a x}$ & $\begin{array}{l}x \text {-directional average absolute } \\
\text { acceleration }\end{array}$ \\
\hline 13.85 & $\mu_{a y}$ & $\begin{array}{l}y \text {-directional average absolute } \\
\text { acceleration }\end{array}$ \\
\hline 15.1 & $\sigma_{a x}$ & $\begin{array}{l}\text { Standard deviation of } x \text {-directional } \\
\text { acceleration }\end{array}$ \\
\hline 15.7 & $\mu_{\text {axy }}$ & Average absolute acceleration \\
\hline 16.625 & $\mu_{p}$ & Average pen pressure $=\operatorname{mean}\{p\}$ \\
\hline 18.075 & $\sigma_{p h}$ & $\begin{array}{l}\text { Standard deviation of the phase }= \\
\text { std }\{P h\}\end{array}$ \\
\hline 18.45 & $p_{\text {med }}$ & $\begin{array}{l}\text { Median of writing pen pressure } \\
=\text { median }\{p\}\end{array}$ \\
\hline 18.575 & $E_{S S}$ & $\begin{array}{l}\text { Entropy of shape signature } \\
\text { function }\end{array}$ \\
\hline 18.65 & $\sigma_{a x y}$ & Standard deviation of acceleration \\
\hline 18.775 & $T_{t}$ & $\begin{array}{l}\text { Duration of the complete writing } \\
\text { process in ms }\end{array}$ \\
\hline
\end{tabular}

Out of 51 scores, 10 common selected scores using mRMR method is shown in Table 2.
Table 2: 10 common selected functional scores

\begin{tabular}{|l|l|l|}
\hline $\begin{array}{l}\text { Average } \\
\text { Rank }\end{array}$ & $\begin{array}{l}\text { Feature Name and } \\
\text { Description. }\end{array}$ & Matching Method \\
\hline 13.325 & X directional & Correlation efficient \\
\hline 13.675 & Pressure Function & Euclidean Distance \\
\hline 13.875 & Absolute acceleration & Correlation Coefficient \\
\hline 13.875 & Pressure function & DTW Distance \\
\hline 15.575 & Y directional & Correlation Coefficient \\
\hline 16.4 & Absolute acceleration & Euclidean Distance \\
\hline 17.075 & Pressure function & Correlation Coefficient \\
\hline 17.55 & Time function & DTW Distance \\
\hline 17.6 & Time function & Euclidean Distance \\
\hline 19.375 & $\begin{array}{l}\text { Magnitude of change } \\
\text { in XY coordinate }\end{array}$ & Euclidean Distance \\
\hline
\end{tabular}

In global feature and functional score concatenated(48+ $51=99$ ). First 48 features are global features and rest 51 features are scores as explain above. Feature number 63 corresponds to score feature number 15 i.e. Magnitude of overall acceleration with correlation score. Feature number 61 corresponds to $\mathrm{X}$ directional acceleration with correlation score. Similarly feature number 97 corresponds to score feature number 49 i.e. Magnitude of acceleration with Euclidean distance score. Out of 99 (global features and scores respectively concatenated), 10 best selected features and scores method are shown in Table 3 using mRMR method.

\begin{tabular}{|l|l|l|l|}
\hline \multicolumn{4}{|c|}{$\begin{array}{l}\text { Table 3: 10 common selected features and functional } \\
\text { scores (jointly selected). G means global feature and S } \\
\text { means score from local feature. }\end{array}$} \\
\hline $\begin{array}{l}\text { Average } \\
\text { Rank }\end{array}$ & $\begin{array}{l}\text { Feature } \\
\text { No }\end{array}$ & Feature Name & G/S \\
\hline 17.2 & 22 & $\begin{array}{l}\boldsymbol{x} \text {-directional average } \\
\text { absolute acceleration }\end{array}$ & G \\
\hline 24.65 & 23 & $\begin{array}{l}\boldsymbol{y} \text {-directional average } \\
\text { absolute acceleration }\end{array}$ & G \\
\hline 25.9 & 63 & Magnitude acceleration & S \\
\hline 27.075 & 61 & X directional acceleration & S \\
\hline 29.45 & 25 & $\begin{array}{l}\text { Standard deviation of x- } \\
\text { directional acceleration }\end{array}$ & G \\
\hline 30.1 & 24 & $\begin{array}{l}\text { Average absolute } \\
\text { acceleration }\end{array}$ & G \\
\hline 30.875 & 62 & Y directional acceleration & S \\
\hline 31.325 & 86 & Pressure Function & S \\
\hline 32.55 & 69 & Pressure Function & S \\
\hline 34.425 & 97 & $\begin{array}{l}\text { Magnitude of } \\
\text { acceleration }\end{array}$ & S \\
\hline Average & rank has been computed as the mean of ranks \\
\hline
\end{tabular}
corresponding to all 40 users in SVC2004 database.

\section{SUPPORT VECTOR MACHINE}

Online signature verification problem can be put as a two class classification problem. In this problem the goal is to separate the two classes by a function which is induced from the training data. Consider the example in Fig. 3. There are many possible linear classifiers that can separate the data, but 
there is only one that maximizes the margin (maximizes the distance between the nearest data point of each class) as shown by bold line in Fig. 3. This is termed as the optimal separating hyperplane.

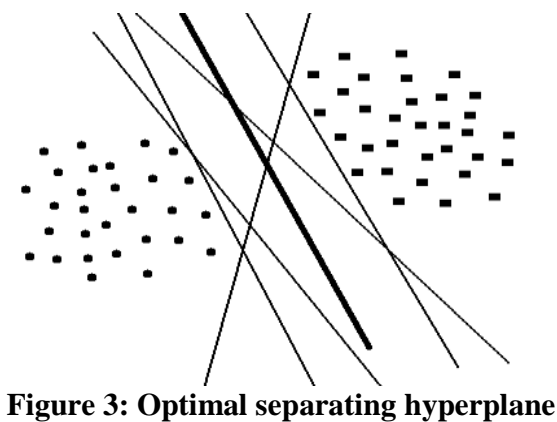

\subsection{The Optimal Separating Hyperplane}

Consider the problem of separating the set of training vectors belonging to two separate classes

$$
\begin{aligned}
& x=\left\{x_{1}, x_{2}, \ldots \ldots, x_{k}\right\} \quad \in \mathcal{R}^{n} \\
& y=\left\{y_{1}, y_{2}, \ldots \ldots, y_{k}\right\} \quad \in\{-1,1\}
\end{aligned}
$$

with a hyperplane: $\quad w^{T} x+b=0$.

The separating hyperplane is said to be optimum if the distance between the closest vectors to the hyperplane is maximal and the separation is without error. There is some redundancy in Eq. 3, and it is appropriate to consider a canonical hyperplane [25-26], where the parameters $w, b$ are given by,

$$
\min _{i}\left|w^{T} x_{i}+b\right|=1
$$

A separating hyperplane in canonical form must satisfy the following constraints,

$$
y_{i}\left[w^{T} x_{i}+b\right] \geq 1, \quad i=1, \ldots \ldots, k
$$

The distance $d(w, b ; x)$ of a point $\mathrm{x}$ from the hyperplane $(w, b)$ is,

$$
d\left(w, b ; x_{i}\right)=\frac{\left|w^{T} x_{i}+b\right|}{\|w\|}
$$

The optimal hyperplane is given by maximizing the margin, $\rho$, subject to the constraints of Eq. 5. The margin is given by,

$$
\begin{aligned}
& \rho(w, b)=\min _{x_{i}: y_{i}=-1} d\left(w, b ; x_{i}\right)+\min _{x_{i}: y_{i}=1} d\left(w, b ; x_{i}\right) \\
& =\min _{x_{i}: y_{i}=-1}\left[\frac{\left|w^{T} x_{i}+b\right|}{\|w\|}\right]+\min _{x_{i}: y_{i}=1}\left[\frac{\left|w^{T} x_{i}+b\right|}{\|w\|}\right] \\
& =\frac{1}{\|w\|}\left(\min _{x_{i}: y_{i}=-1}\left|w^{T} x_{i}+b\right|+\min _{x_{i}: y_{i}=1}\left|w^{T} x_{i}+b\right|\right) \\
& =\frac{2}{\|w\|}
\end{aligned}
$$

The maximization of $\rho$ can be implemented by minimizing a function $\phi(w)$, where

$$
\phi(w)=\frac{1}{2}\|w\|^{2}
$$

It is independent of $b$ because provided Eq. 5 is satisfied (i.e. it is a separating hyperplane) changing $\mathrm{b}$ will move it in the normal direction to itself. Accordingly the margin remains unchanged but the hyperplane is no longer optimal in that it will be nearer to one class than the other. The saddle point of the Lagrange functional gives the solution to the optimization problem of Eq. 8 under the constraints of Eq. 5 [27].

$$
\phi(w, b, \alpha)=\frac{1}{2} w^{T} w-\sum_{i=1}^{k} \alpha_{i}\left(y_{i}\left[w^{T} x_{i}+b\right]-1\right)
$$

where $\alpha$ are the Lagrange multipliers. The Lagrangian has to be minimised with respect to $w, b$ and maximised with respect to $\alpha \geq 0$. Lagrangian duality enables the primal problem, Eq. 9 , to be transformed to its dual problem, which is easier to solve. The dual problem is given by

$$
{ }_{\alpha}^{\max } W(\alpha)={ }_{\alpha}^{\max }\left(\begin{array}{l}
\min \\
w, b
\end{array} \phi(w, b, \alpha)\right) .
$$

Thus, differentiating $\phi(w)$ with respect to $w, b$ and setting the results equal to zero, we get the following two conditions of optimality:

$$
\begin{array}{ll}
\text { Condition 1: } & \frac{\partial \phi}{\partial w}=0 \Rightarrow w=\sum_{i=1}^{k} \alpha_{i} y_{i} x_{i} \\
\text { Condition 2: } & \frac{\partial \phi}{\partial b}=0 \Rightarrow \sum_{i=1}^{k} \alpha_{i} y_{i}=0
\end{array}
$$

The solution vector $w$ is defined in terms of an expansion that involves the $k$ training examples. Although this solution is unique by virtue of the convexity of the Lagrangian, the same can not be said about the Lagrange coefficients, $\alpha_{i}$.

It is also important to note that at the saddle point, for each Lagrange multiplier $\alpha_{i}$, the product of that multiplier with its corresponding constant vanishes, as shown by

$$
\left[\alpha_{i} y_{i}\left(w^{T} x_{i}+b\right)-1\right]=0 \text { for } i=1,2, \ldots, k
$$

Therefore those multipliers exactly meeting Eq. 12 can assume nonzero values. This property follows from the KuhnTucker conditions of optimization theory [28]. Hence only the points $x_{i}$ which satisfy,

$$
y_{i}\left(w^{T} x_{i}+b\right)=1
$$

will have non-zero Lagrange multipliers. These points are termed Support Vectors (SV). If the data is linearly separable all the SV will lie on the margin and hence the number of SV can be very small. Consequently the hyperplane is determined by a small subset of the training set. The other points could be removed from the training set and recalculating the hyperplane would produce the same answer [25]. 


\subsection{Gamma and C in SVM}

In signature verification number of observation is very less compared to number of features. It is better to use the linear SVM rather than nonlinear kernel SVM. Because the number of features is already much larger than the number of observations, non linear mapping is not essential which map the data into a higher dimensional features space. If linear SVM is used then only parameter $\mathrm{C}$ is needed to search for the better accuracy.

As conjectured in [29] a small C yields a high error rate on the training patterns, whereas a large $\mathrm{C}$ is bound to result in a high error rate on future patterns. In this signature verification problem training accuracy is achieving $100 \%$ with large $\mathrm{C}$ but testing accuracy is not able to reach near $100 \%$. So to get better testing accuracy and thereby a more reliable SVM classifier it is needed to restrict the value of parameter $\mathrm{C}$ within a limited range.

\section{NUMERICAL EXPERIMENT}

Six numerical set up has been consider for verification using SVM as shown in Table 4

Table 4: Six numerical setups.

\begin{tabular}{|c|l|l|}
\hline Setup 1: & $\begin{array}{l}\text { Global Feature Based Verification Using } \\
\text { Nonlinear (RBF kernel) SVM }\end{array}$ \\
\hline Setup 2: & $\begin{array}{l}\text { Global Feature Based Verification Using Linear } \\
\text { SVM }\end{array}$ \\
\hline Setup 3: & $\begin{array}{l}\text { Local Score Based Verification Using Nonlinear } \\
\text { (RBF kernel) SVM }\end{array}$ \\
\hline Setup 4: & $\begin{array}{l}\text { Local Score Based Verification Using Linear } \\
\text { SVM }\end{array}$ \\
\hline Setup 5: & $\begin{array}{l}\text { Global Feature and Local Score Based } \\
\text { Verification Using Nonlinear (RBF kernel) SVM }\end{array}$ \\
\hline Setup 6: & $\begin{array}{l}\text { Global Feature and Local Score Based } \\
\text { Verification Using Linear SVM }\end{array}$ \\
\hline
\end{tabular}

The LIBSVM- A Library for Support Vector Machines toolbox is used for experiment [30]. Since version 1.2, it implements an SMO-type algorithm proposed in [31]. All the algorithms proposed in this thesis are implemented in MATLAB 7.7 [32]. Two class SVM parameters and their ranges are shown in Table 5 .

Table 5: Parameter for the two class SVM classifier

\begin{tabular}{rlr}
\hline Parameter Name & Value \\
\hline Kernel Type & Radial basis function (RBF) \\
& $K\left(x_{i}, x\right)=e^{-\gamma\left\|x_{i}-x\right\|^{2}}$ \\
Degree & 0 \\
Gamma $(\gamma)$ & $2^{-60}: 2^{-0.5}: 2^{10}$ \\
Coefficient & 0 \\
$C$ & $2^{-50}: 2^{-0.5}: 2^{30}$ \\
Cache size & 50 \\
epsilon & 0.001 \\
SVM type & Two Class c SVM \\
\hline
\end{tabular}

\section{RESULTS}

The results from the final stage of signature verification using SVM is shown in Table 6. User specific ranked features are used in this experiment. Summary of results using SVM with common ranked features is shown in Table 7.

Table 6: Summary of results using SVM technique and user specific ranked feature

\begin{tabular}{|c|c|c|c|c|c|}
\hline Setup & $\begin{array}{c}\text { \% } \\
\text { Training } \\
\text { Accuracy }\end{array}$ & $\begin{array}{c}\% \\
\text { Testing } \\
\text { Accuracy }\end{array}$ & $\log _{2} \gamma$ & $\log _{2} \boldsymbol{C}$ & $\begin{array}{c}\text { Time } \\
(\mathbf{s})\end{array}$ \\
\hline 1 & 100 & 95.75 & -52.05 & -27.3 & 0.39 \\
\hline 2 & 99.88 & 95.94 & NA & -25.33 & 0.34 \\
\hline 3 & 100 & 91.75 & -45.91 & -22.25 & 0.47 \\
\hline 4 & 100 & 91.5 & NA & -16.2 & 0.22 \\
\hline 5 & 100 & 96 & -55.91 & -40.98 & 0.41 \\
\hline 6 & 100 & 97.69 & NA & -34.58 & 0.22 \\
\hline
\end{tabular}

Table 7: Results using SVM and common ranked features

\begin{tabular}{|c|l|l|l|l|l|}
\hline $\begin{array}{r}\text { Setu } \\
\mathrm{p}\end{array}$ & $\begin{array}{c}\text { \% } \\
\text { Training } \\
\text { Accuracy }\end{array}$ & $\begin{array}{c}\text { \% } \\
\text { Testing } \\
\text { Accuracy }\end{array}$ & $\log _{2} \boldsymbol{\gamma}$ & $\log _{2} \boldsymbol{C}$ & $\begin{array}{c}\text { Time } \\
(\mathbf{s})\end{array}$ \\
\hline 1 & 100 & 91.57 & -27.88 & -7.83 & 0.39 \\
\hline 2 & 99.63 & 90.69 & NA & -23.79 & 0.74 \\
\hline 3 & 100 & 90.75 & -27.8 & -8.62 & 0.36 \\
\hline 4 & 100 & 89.5 & NA & -17.11 & 0.26 \\
\hline 5 & 100 & 92.13 & -28.95 & -26.9 & 0.37 \\
\hline 6 & 100 & 92.38 & NA & -22.39 & 0.26 \\
\hline
\end{tabular}

Time indicated in Table 6 and 7 is the average time required to train 20 signatures and to test 40 signatures.ANN classifier model is formed with one hidden layer consist of five neuron feed-forward back propagation network. Average Testing accuracy is $96.125 \%$ for user specific best selected 5 features. Average Testing accuracy drops down to $87.25 \%$ for best selected common 5 features.

\section{DISCUSSION AND CONCLUSION}

Acceleration and pressure related features are mostly selected in all the ranking processes. By observing the performance of linear and nonlinear SVM it is found that linear SVM with only six number of features gives better result than non linear SVM (with RBF kernel function). Training accuracy is $100 \%$ in the case of nonlinear SVM but testing accuracy is not close to training accuracy. But in the case of linear SVM testing accuracy is closer to the training accuracy. It implies that linear SVM is more reliable in global feature based verification. Time taken in linear SVM is also less. All these results indicate that linear SVM outperforms the nonlinear (RBF kernel) SVM for this particular case. Combination of global features and functional scores is proven to be fruitful giving better verification accuracy $(97.69 \%)$. 


\section{ACKNOWLEDGMENTS}

The authors would like to thank the authorities of IIT Kharagpur and EU-India CultureTech Project under ECCP for their support to carry out this research work.

\section{REFERENCES}

[1] Jain, Anil K., Griess, Friederike D. and Connell, Scott D. 2002. On-line signature verification, Pattern Recognition, vol. 35 , no. 12 , pp. $2963-2972$.

[2] Herbst, N. M. and Liu, C. N. 1977. Automatic signature verification based on accelerometry, IBM J. Res. Dev., vol. 21, pp. 245-253.

[3] Impedovo, D. and Pirlo, G. 2008. Automatic signature verification: The state of the art, IEEE Trans. Syst., Man, Cybern. C, Appl. Rev., vol. 38, no. 5, pp. 609-635.

[4] Leclerc, F. and Plamondon, R. 1994. Automatic signature verification: the state of the art-1989-1993, Int. J. Pattern Recognition Artif. Intel., vol. 8, no. 3, pp. 643-660.

[5] Plamondon, R. and Lorette, G. 1989. Automatic signature verification and writer identification - the state of the art, Pattern Recognition, vol. 1, no. 2, pp. 107131.

[6] Sabourin, R., Genset, G. and Preteux, F. 1997. Off-Line Signature Verification by Local Granulometric Size Distribution, IEEE Trans. Pattern Analysis and Machine Intelligence, vol. 19, no. 9, pp. 976-988.

[7] Aguilar, J.F., Krawczyk, S., Garcia, J.O. and Jain, A.K. 2005. Fusion of Local and Regional Approaches for OnLine Signature Verification, Proc. Int'l Workshop Biometric Recognition System, pp. 188-196.

[8] Bajaj. R. and Chaudhary, S. 1997. Signature Verification Using Multiple Neural Classifiers, Pattern Recognition, vol. 30, pp. 1-87.

[9] Van, B. Ly, Garcia-Salicatti, S. and Dorizzi, B. 2007 On using the Viterbi path along with HMM likelihood information for online signature verification, IEEE Trans. Syst., Man, Cybern. B, Cybern., vol. 37, no. 5, pp. 1237-1247.

[10] Alister, K. and Yanikoglu, B. 2005. Identity Authentication Using Improved On-Line Signature Verification Method, PRL, vol. 26, no. 18, pp. 24002408 .

[11] Justino, E. J. R., Bortolozzi, F. and Sabourin, R. 2005. A comparison of SVM and HMM classifiers in the offline signature verification, PRL, vol. 26, pp. 1377-1385.

[12] Feng, H. and Wah, C. C. 2003. Online signature verification using a new extreme points warping technique, PRL vol. 24, pp. 2943-2951.

[13] Munich, M. E. and Perona, P. 2003. Visual identification by signature tracking, IEEE Trans. PAMI, vol. 25, no. 2, pp. $200-217$.

[14] Fang, P., Wu, Z., Shen, F., Ge, Y. and Fang, B. 2005. Improved DTW algorithm for online signature verification based on writing forces, in Proc. ICIC-Part I, vol. 3844, Berlin, Germany, pp. 631-640.

[15] Kholmatov A. and Yanikoglu, B. 2005. Identity authentication using improved online signature verification method, PRL, vol. 26, no. 15, pp. 2400 2408.

[16] Faúndez-Zanuy, M. 2007. On-line signature recognition based on VQ-DTW, Pattern Recognition, vol. 40, no. 3, pp. 981-992.

[17] Lei, H. and Govindaraju, V. 2005. A comparative study on the consistency of features in on-line signature verification, PRL vol. 26, no. 15, pp. 2483-2489.

[18] Yeung, D., Chang, H., Xiong, Y., George, S., Kashi, R., Matsumoto, T. and Rigoll, G. 2004. SVC 2004: First international signature verification competition, in Proc. ICBA, vol. 3072, LNCS, D. Zhang and A. K. Jain, Eds., Berlin, Germany, 2004, pp. 16-22. [Online]. Available: http://www.cse.ust.hk/svc2004/ results.html

[19] Zhang, K., Pratikakis, I., Cornelis, J. and Nyssen, E. 2000. Using landmarks to establish a point-to-point correspondence between signatures, Pattern Anal. Appl., vol. 3, no. 1, pp. 69-75.

[20] Nalwa, V.S. 1997. Automatic On-line Signature Verification, in Proceedings of the IEEE, vol. 85, pp.215-239.

[21] Martens, R. and Claesen, L. 1998. Incorporating local consistency information into the online signature verification process, Int. J. Doc. Anal. Recognit. (IJDAR), vol. 1, no. 2, pp. 110-115.

[22] Peng, H., Long, F., and Ding, C. 2005. Feature selection based on mutual information: Criteria of maxdependency, max-relevance, and min-redundancy, IEEE Trans. PAMI, vol. 27, no. 8, pp. 1226-1238.

[23] Duda, R. O., Hart, P. E. and Stork, D. G. 2006. Pattern Classification, Wiley India Edition, 2nd Edition.

[24] Ding, C. and Peng, H.C. 2003. Minimum Redundancy Feature Selection from Microarray Gene Expression Data, Proc. of second IEEE CSBC, pp. 523-528.

[25] Vapnik, V. 1995. The Nature of Statistical Learning Theory, Springer Verlag, New York.

[26] Kantardzic, M. 2011. Data Mining: Concepts, Models, Methods, and Algorithms- $2^{\text {nd }}$ edition John Wiley and sons.

[27] Cortes, C. and Vapnik, V. 1995. Support vector networks. Machine Learning, 20:273 - 297.

[28] Bertsekas, D. P. 1999. Nonlinear Programming, 2nd ed. Belmont, MA: Athena Scientific.

[29] Scholkopf, B. and Smola, A. J. 2002. Learning with Kernels, MIT Press.

[30] Chang, C. C. and Lin, C. J. 2001. LIBSVM: A library for support vector machines, Tech. Rep., [Available]. Online: http://www.csie.ntu.edu.tw/»cjlin/libsvm.

[31] Fan, R. E., Chen, P. H. and Lin, C. J. 2005. Working set selection using the second order information for training svm, Journal of Machine Learning Research, vol. 6, no. 12, pp. $1889-1918$

[32] MATLAB, User's Guide, The MathWorks, Inc., 1994 2009, Version 7.7.0.471 (R2008b) http://www.mathworks.com. 